Received June 20, 1972.

1 Brown, G. M., and Peckett, A., Nature, 234, 262 (1971).

2 O'Hara, M. J., Biggar, G. M., Richardson, S. W., Ford, C. E., and Jamieson, B. G., Proc. Apollo 11 Lunar Sci. Conf. Geochim. Cosmochim. Acta Supp. 1, 1, 695 (1970).

3 Biggar, G. M., O'Hara, M. J., Peckett, A., and Humphries, D. J., Proc. Second Lunar Sci. Conf., 1, 853 (M.I.T. Press, Massachusetts, 1971).

4 Ringwood, A. E., and Green, D. H., Earth Planet. Sci. Lett., 14, 14 (1972)

5 Ringwood, A. E., J. Geophys. Res., 75, 6453 (1970).

6 Green, D. H., Ringwood, A. E., Ware, N. G., Hibberson, W. O., Major, A., and Kiss, E., Proc. Second Lunar Sci. Conf., 1, 601 (M.I.T. Press, Massachusetts, 1971).

7 Green, D. H., Ringwood, A. E., Ware, N. G., and Hibberson, W. O., Proc. Third Lunar Sci. Conf., 1 (in the press).

\section{Optical Polarization of the Supernova in NGC 5253}

IF a type I supernova is the result of the explosion of a star with a degenerate nucleus (see, for example, Baglin ${ }^{1}$ ) it is likely that a large magnetic field will be present in the radiating region for a short period following the outburst. Depending on the emission process a sufficiently large field could produce a measurable optical circular polarization. For example, for bremsstrahlung in an optically thick but very dilute plasma in an ordered magnetic field of $H$ gauss, the fractional circular polarization in the optical continuum is $q \sim 10^{-9} H$ (see refs. 2 and 3). If the degenerate progenitor of the supernova is entirely destroyed in the explosion, conservation of magnetic flux dictates that $q$ would reach the limit of detectability $\left(q_{\text {LIM }} \sim 3 \times 10^{-6}: H_{\text {LIM }} \sim 3,000 \mathrm{G}\right)$ in only $20 \mathrm{~s}$ for either a white dwarf (initial radius $r_{0} \sim 10^{9} \mathrm{~cm}$, initial field $H_{0} \sim 10^{6}$ G) or a neutron star $\left(r_{0} \sim 10^{6} \mathrm{~cm}, H_{0} \sim 10^{12} \mathrm{G}\right)$ assuming an expansion velocity of $10^{4} \mathrm{~km} \mathrm{~s}^{-1}$. If a sizable fragment survives, however, a measurable polarization might be seen for a much longer period. For synchrotron emission smaller fields can produce a detectable polarization. According to Legg and Westfold ${ }^{4}$ a hypothetical field $H \sim 10^{6} \mathrm{G}$ in the Crab nebula would produce $q \sim 10 \%$ in the visible: since $q \propto H^{1 / 2}$ a polarization $q_{\text {LIM }} \sim 3 \times 10^{-6}$ corresponds to $H_{\text {LIM }}$ $\sim 10^{-3} \mathrm{G}$. However, the precise conditions strongly influence ${ }^{5,6}$ the relations between $q$ and $H$; the interpretation would therefore be uncertain even if this mechanism were shown to be dominant. The influence of radiative transfer in the dense layers near the star and of a possible fluorescence emission in the outer layers of the shell ${ }^{7}$ would make the interpretation for the synchrotron process even more difficult.

We have measured the polarization of the bright supernova discovered by $\mathrm{Kowal}^{8}$ on May 13, 1972, in the irregular galaxy NGC 5253. Spectroscopic evidence ${ }^{9,10}$ suggests that it is type I: an earlier supernova ( $Z$ Centauri) in this galaxy obsarved in 1895 was also type I. The fractional linear and circular polarizations measured with no filter $(\lambda \lambda 3500$ to $6500 \AA$ ) on June 13.3 (UT), 1972, are respectively: $p=$ $(3.5 \pm 2.0) \times 10^{-3}, q=-(0.8 \pm 0.4) \times 10^{-4}$. The linear polarization is comparable with the value measured by Serkowski ${ }^{11}$ in the supernova in NGC 5253. An explanation for the small upper limit on the circular polarization $\left(|q|<10^{-4}\right)$ may be sought in terms of either (1) bremsstrahlung or synchrotron radiation, followed by radiative transfer and fluorescence, or (2) interstellar circular polarization ${ }^{12}$. It is unlikely that the latter is involved, because if we assume that the observed linear polarization $p=0.35 \%$ is produced by aligned grains in our Galaxy, then the expected broad band circular polarization is too small, namely $q \simeq 0.7 \times 10^{-5}$. (This conclusion is based on data for 6 stars studied by Kemp and Wolstencroft (unpublished).) The upper limits to $H$ for the bremsstrahlung and synchrotron processes obtained from $i q \mid \leq 10^{-4}$ are $10^{5} \mathrm{G}$ and $1 \mathrm{G}$ respectively. (The former value includes allowance for radiative transfer ${ }^{3}$.) If either of these processes produced the emission close to the star, then the field was less than $10^{5} \mathrm{G}$ at the time of our observation.

R. D. WOLSTENCROFT

J. C. KEMP*

Institute for Astronomy,

University of Hawaii, Honolulu, Hawaii

Received July 10, 1972.

* On leave from the Department of Physics, University of Oregon, Eugene, Oregon 97403.

1 Baglin, A., Astrophys. Lett., 1, 143 (1968).

2 Kemp, J. C., Astrophys. J., 162, 169 (1970).

${ }^{3}$ Gnedin, Y. N., Dolginov, A. Z., and Silant'ev, N. A., Zh. Eksper $i$ Teoret. Fiz, 59, 865 (1970) (English transl., Soviet Phys.JETP, 32, 472 (1971)).

${ }^{4}$ Legg, M., and Westfold, K., Astrophys. J., 154, 499 (1968).

5 Pacholczyk, A., and Swihart, T., Astrophys. J., 161, 415 (1970).

6 Melrose, D. B., Astrophys. Lett., 8, 35 (1971).

7 Morrison, P., and Sartori, L., Astrophys. J., 158, 541 (1969).

8 Kowal, C. T., IAU Circular No. 2406 (1972).

9 Herbig, G. H., IAU Circular No. 2408 (1972).

10 Stephenson, C. B., IAU Circular No. 2408 (1972).

11 Serkowski, K., Astrophys. J., 160, 1083 (1970).

12 Kemp, J. C., Astrophys. J., 175, L35 (1972).

\section{Crust and Mantle of the Gulf of Mexico}

A SEEMING paradox has puzzled investigators of the crustal structure of the Gulf of Mexico since Ewing et al. ${ }^{1}$ calculated that a unit area of the rather thick crust in the gulf contains less mass than does a combination of the crust and enough of the upper mantle to make a comparable thickness in the Atlantic Ocean. They also noted that the free-air gravity of the gulf is essentially normal and fails by a large factor to be low enough to reflect the mass difference that they calculated. We propose a solution to this problem.

The study by Ewing et al. was based on comparative summations of thickness multiplied by density for the various layers in each setting. Thickness was derived from seismic-refraction inflexions and density from Nafe and Drake's curve ${ }^{2}$ relating density and seismic velocity. From this crustal analysis and data for the Gulf of Mexico's Sigsbee Deep and the western Atlantic basin ${ }^{1}$, the present floor of the gulf should lie at a depth of only $1.9 \mathrm{~km}$, whereas its actual depth is $3.7 \mathrm{~km}$.

Wilhelm and Ewing ${ }^{3}$ suggest that oceanization may explain an increased crustal density for the Gulf of Mexico. This concept, developed by Beloussov ${ }^{4}$, is that mantle material intrudes crust of the continental type, expels volatile components, and converts silicate minerals into denser phases. Crustal products created by such metasomatism, however, should be compatible with the Nafe-Drake curve, so the concept of oceanization does not explain the mass deficiency.

Antoine and Pyle ${ }^{5}$ suggest that turbidity-current deposits in the gulf might have a relatively high density for their seismic velocity, thereby causing the Nafe-Drake relation to give incorrect densities for this area. But, as Menard ${ }^{6}$ points out, the densities required to make the crust of the Gulf of Mexico match the section in the Atlantic Ocean fall outside the entire field of Nafe-Drake data points, which include a wide variety of hard rocks and sediments. Menard concludes that the compensating mass required to provide the balance must lie in the mantle of the Gulf of Mexico. The density of the mantle in the gulf was not included in the calculations of Ewing et al. ${ }^{1}$, as they considered it to be the same as that of the Atlantic basin.

We propose that the differences are caused partly by differences in depth to the low-velocity interface, which lies in the mantle at a depth of about $60 \mathrm{~km}$, and chiefly by small temperature differences in the thick layer of mantle above the interface. Owing to sediment loading, the Mohorovičic (M) discontinuity (the top of the mantle) lies at a depth of $18.8 \mathrm{~km}$ under the Sigsbee Deep, or about $6 \mathrm{~km}$ deeper than in the western Atlantic Ocean. Some of the compensating mass is 